\title{
Micro-usinage par laser pour interconnexions micro-électroniques
}

\author{
P. Morillon, J.-Cl. Farcy, F. Gilbert, G. Hélias et G. Legeay
}

SAT/SII-T, BP. 130, 22103 Dinan, France

\begin{abstract}
Résumé: Le micro-perçage par laser des vias entre niveaux conducteurs des modules multipuces (MCM) permet un saut technologique sans augmentation des coûts. Un laser $\mathrm{CO}_{2}$ TEA à impulsion longue a été dẻveloppé pour percer les diélectriques couches épaisses. Un laser excimère est utilisé pour percer les diélectriques organiques couches minces, nécessaires aux futurs MCM rapides.
\end{abstract}

\begin{abstract}
Laser micro-drilling of vias between conductors levels in MultiChips Modules (MCM) is a technological breakthrough with no extra costs added. $\mathrm{A} \mathrm{CO}_{2}$ TEA laser with widened pulse was designed to drill vias through thick films dielectrics. An excimer laser is used to drill thin films organic dielectrics, witch are mandatory for the future fast working MCM.
\end{abstract}

\section{INTRODUCTION}

\subsection{Les MCM}

La micro-électronique hybride a été développée dans notre société depuis les années 70 pour répondre aux besoins des matériels de télécommunication puis des matériels militaires et, plus récemment, du marché libre. Les modules multipuces (MCM) représentent le haut de gamme en termes de densité de circuit et de fréquence de travail. Ces circuits à forte densité de composants silicium en surface sont multicouches (fig. 1).

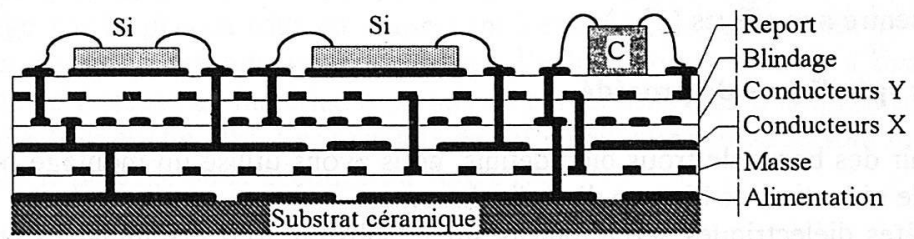

fig.1: Structure type d'un MCM.

Pour les réaliser, deux filières technologiques principales sont mises en oeuvre: La filière technologique "sérigraphie-couches épaisses" est utilisée pour les MCM actuels. La filière "couches minces" (qui fait appel également à la photolithographie) est envisagée pour les futurs MCM rapides (horloges à plus de $300 \mathrm{Mhz}$ ). 


\subsection{Un problème commun: les vias}

Dans l'évolution des technologies classiques, il est apparu que les vias entre niveaux conducteurs limitaient la densité des circuits:

La technologie sérigraphie - couches épaisses est purement additive, les conducteurs sont implantés à un pas de 120 à $400 \mu \mathrm{m}$. Cependant, les vias réalisés par sérigraphie directe ont un diamètre minimum de $200 \mu \mathrm{m}$.

La technologie couches minces (avec diélectrique organique) est le plus souvent soustractive. Les épaisseurs de couches vont de 0,1 à $20 \mu \mathrm{m}$, les conducteurs et les vias sont réalisés par photolithographie. Typiquement les conducteurs des MCM ont une largeur de 20 $\mu \mathrm{m}$, alors que les vias dans les polymères sont photogravés au diamètre minimum de $40 \mu \mathrm{m}$.

De plus, les procédés traditionnels de réalisation des vias, nécessitant de nombreuses étapes, limitaient également la productivité. C'est pourquooi l'étude du micro-perçage de vias par laser dans les deux filières technologiques a fait partie de la contribution SAT au projet Eureka n462 «PEPITE».

\section{PERCAGE DES VIAS COUCHES EPAISSES PAR LASER $\mathrm{CO}_{2}$}

\subsection{Sélection du type de laser}

Dans cette filière les épaisseurs de couches vont de 8 à $50 \mu \mathrm{m}$, les diélectriques entre les niveaux de conducteurs sont, après cuisson, des matériaux totalement inorganiques. Pour rester compatible avec les autres étapes technologiques, le perçage des vias dans les diélectriques doit réaliser des trous borgnes avec un arrêt sélectif sur le conducteur inférieur (typiquement en or pour ce type de produit).

Le choix d'un laser $\mathrm{CO}_{2}$ pour l'application couches épaisses s'est appuyé sur des éléments de bibliographie, sur le savoir faire de la société, et sur quelques essais préliminaires:

Pour les diamètres de trous visées, de l'ordre de $50 \mu \mathrm{m}$, l'optique autorise l'emploi de lasers de toutes les longueurs d'ondes (selon le critère pratique: diamètre de l'image supérieur à 3 longueurs d'ondes).

La grande réflectivité dans l'infrarouge des métaux utilisés (fig. 2) assure la sélectivité recherchée.

Enfin, les premiers essais nous ont montré une dégradation des métallisations de fond de trou avec un laser YAG et une faisabilité très probable en Excimère et en $\mathrm{CO}_{2}$. Par contre, le perçage Excimère de diélectriques minéraux conduisait à un coût d'exploitation inabordable dans cette filière (vitesses d'ablation trop faibles).

Le laser $\mathrm{CO}_{2}$ nous a alors semblé l'outil adapté pour ce travail, c'est sur lui que nous avons concentré nos efforts [1].

\subsection{Etudes spécifiques de procédé}

Pour obtenir des bords de trous bien définis, nous avons utilisé un montage optique de projection et de réduction de l'image d'un diaphragme.

Les pâtes diélectriques sérigraphiées sur le substrat sont manipulables après un simple séchage avant cuisson. Il a été remarqué que les liants organiques encore présents dans la pâte à ce stade favorisent l'absorption du rayonnement IR. Un seul tir est alors suffisant pour percer toute la gamme des épaisseurs usuelles. 


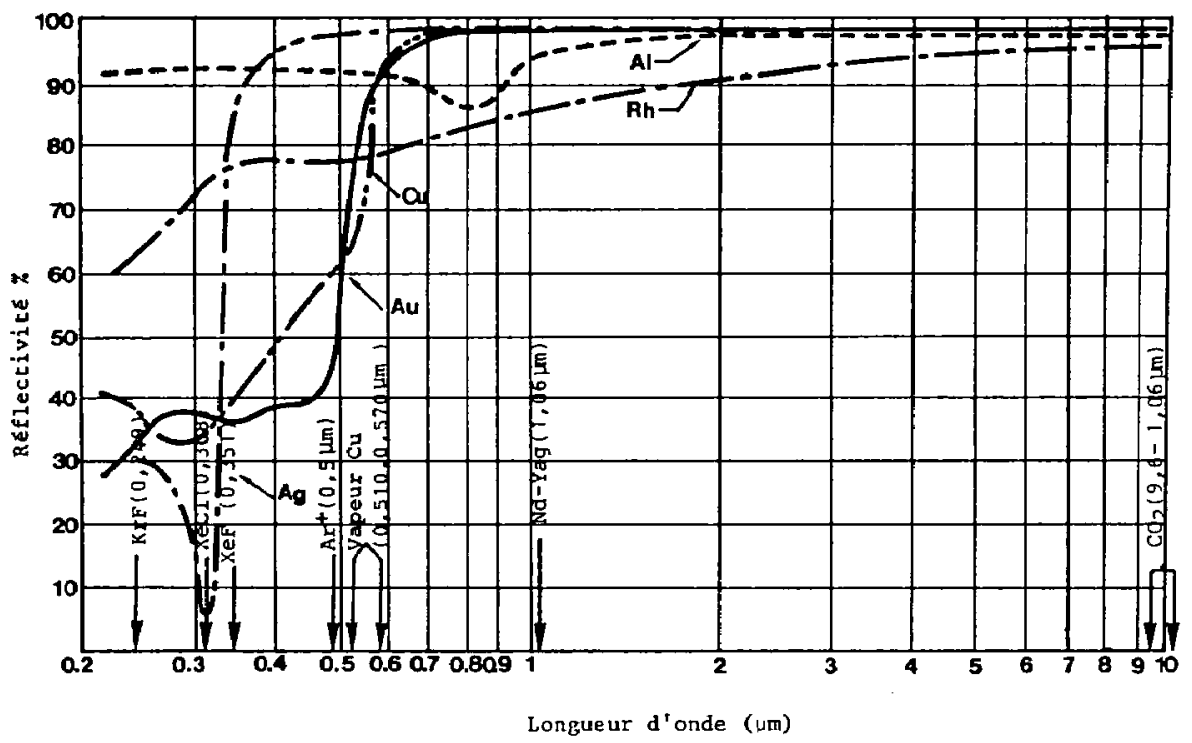

figure 2: Réflectivité de films minces métalliques de l'UV à l'IR et longueurs d'ondes de quelques lasers courants [2].

Cependant, nous avons rapidement noté la présence dans les trous de billes de verre refondues, difficilement acceptables pour la suite des opérations. L'utilisation d'un laser $\mathrm{CO}_{2}$ TEA traditionnel conduisait à des résultats dispersés selon les références de pâtes plus ou moins riches en verre (fig. 4a). Nous avons alors orienté nos investigations dans deux directions:

- Sélection de pâtes diélectriques peu riches en verre.

- Optimisation du couplage de l'énergie du laser.

Enfin, l'opération suivante de remplissage des vias de petite taille par de la pâte conductrice a nécessité également une mise au point.

\subsection{Travaux sur le couplage faisceau / matériau}

Des modifications de la source laser TEA ont permis d'étaler l'impulsion sur environ $10 \mu \mathrm{s}$ (au lieu de quelques centaines de nanosecondes) (fig. 3). On a ainsi supprimé le phénomène connu d'écrantage par le plasma tout en conservant l'essentiel des propriétés de l'ablation laser rapide: pas de résidu refondu ou calciné, pas d'échauffement notable à distance. Le meilleur couplage du faisceau au matériau a permis de percer sans résidu la quasi-totalité des références de diélectriques (fig. $4 \mathrm{~b}$ ). Les résultats de cette nouvelle source se sont même révélés excellents dans le perçage ou la découpe du verrre [3].

\subsection{Développement d'une machine}

Une machine de micro-perçage a été construite pour exploiter le procédé dans des conditions de production [4]. Elle est capable de percer 25 trous par seconde en "stop and go" avec une précision de placement de $\pm 5 \mu \mathrm{m}$. 


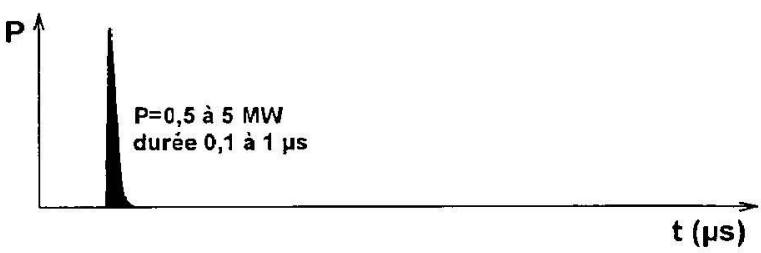

Plasma très important

Peu d'interaction / matériau Légère vaporisation Pas d'effet thermique

$t(\mu s)$

figure 3-a: Laser TEA

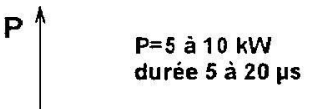

figure 3-b: Laser TEA à impulsion longue

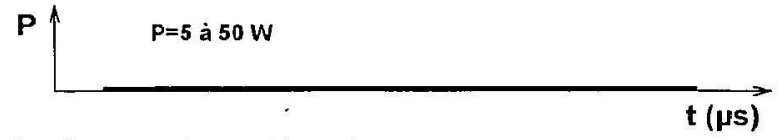

Effet thermique prépondèrant

figure 3-c: Laser continu ou découpé

\section{Plasma très réduit \\ Vaporisation (prépondérant) \\ Très peu d'effet thermique}

$t(\mu s)$

$t(\mu s)$

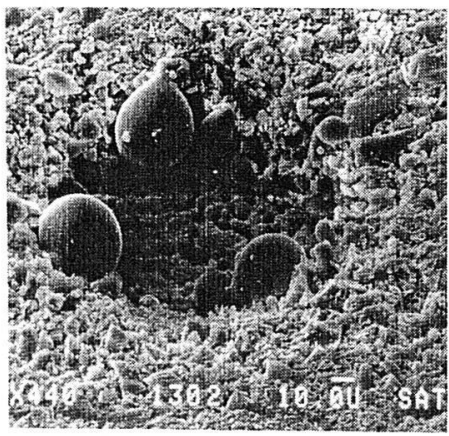

a)

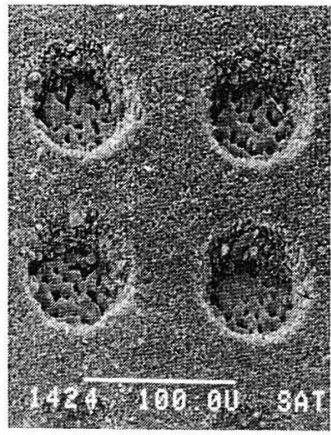

b)

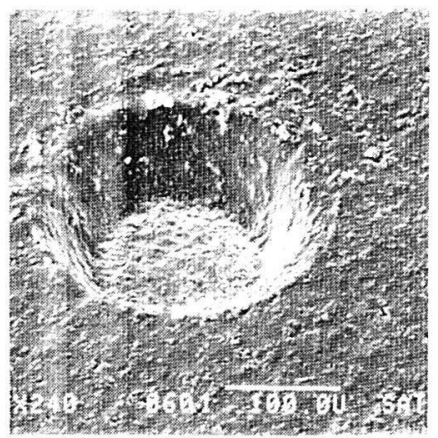

c)

figare 4: Trous percés dans une pâte diélectrique, a) pâte crue. laser $\mathrm{CO}_{2}$ TEA; b) pâte crue, laser $\mathrm{CO}_{2}$ TEA à impulsion longue; $c$ ) iden $b$ après cuisson.

Les tables $\mathrm{X} / \mathrm{Y}$ sont sur coussin d'air et pilotées par des moteurs linéaires. Un dispositif de mise au point automatique a été prévu pour compenser les dispersions d'épaisseur des substrats posés sur la platine.

Par rapport à l'ancien procédé, l'interfaçage de la machine laser avec la station de CAO permet d'éviter l'exécution d'un masque (souvent sous-traitée), et de supprimer la réalisation d'un écran de sérigraphie. 


\subsection{Résultats}

Le procédé a été validé par des essais climatiques sur des mires (gamme militaire) et nous disposons d'une machine pleinement opérationnelle.

Parallèlement au saut technologique, le perçage laser a permis d'importants gains de productivité: Le nombre d'étapes technologiques nécessaires pour réaliser un niveau de diélectrique est de 7 , à comparer à 12 dans un procédé classique ou à 14 pour une méthode à base de diélectrique photoimageable proposée actuellement sur le marché [5].

Un circuit de type DSP pour traitement d'image en temps réel est en cours de développement. Il comportera des conducteurs de $80 \mu \mathrm{m}$ au pas de $160 \mu \mathrm{m}, 6$ niveaux conducteurs, et plus de 10000 vias.

\section{PERCAGE DES VIAS COUCHES MINCES PAR LASER EXCIMERE}

\subsection{Sélection du type de laser}

La construction de MCM couches minces fait appel à des diélectriques organiques de faible constante diélectrique $(2,7$ à 3,7$)$ pour minimiser les délais de propagation des signaux [6][7]. Les matériaux à percer sont de la classe des polyimides, en épaisseurs de 8 à $20 \mu \mathrm{m}$. Les vias sont des trous borgnes percés sélectivement sur des conducteurs inférieurs métalliques (typiquement en cuivre ou en aluminium) épais de 2 à $5 \mu \mathrm{m}$. L'ouverture visée, de l'ordre de $20 \mu \mathrm{m}$, assure une grande densité de conducteurs tout en garantissant une résistance de contact négligeable entre niveaux successifs.

Des essais préliminaires ont montré que les lasers YAG ou $\mathrm{CO}_{2}$ laissaient une propreté de perçage insuffisante pour l'étape suivante de métalisation (qualité des contacts, adhérence). Les trous percés par Excimère (à $248 \mathrm{~nm}$ comme à $308 \mathrm{~nm}$ ) étaient très propres, les dimensions souhaitées ont été obtenues sans problème, nous avons constaté une sélectivité parfaite sur des couches d'or rugueuses.

\subsection{Etudes de procédé}

Les premiers travaux ont porté sur l'interaction faisceau / diélectriques organiques. On a déterminé les vitesses d'ablation en fonction de la fluence sur différents diélectriques organiques (fig. 5)[8]. Le comportement de tous les produits testés de la classe des polyimides est finalement très voisin, ce qui procure un avantage de "portabilité" pour le procédé.

Si l'on considère les courbes de réflectivité des métaux (fig. 2), le perçage en U.V. ne semble pas favorable à une bonne sélectivité sur le conducteur inférieur. Cependant une étude attentive de la littérature montre que les seuils d'ablation sont très différents suivant les matériaux [9]. Pour les métaux, les seuils de dommages permanents se situent vers $1 \mathrm{~J} / \mathrm{cm}^{2}$ ou plus, dès que leur épaisseur dépasse le micron [8][10]. Or à cette fluence, la vitesse d'ablation des polyimides semble approcher d'un palier (fig.5). On retrouve par ce biais une excellente sélectivité en choisissant une fluence adaptée.

Enfin, la longueur d'onde de $308 \mathrm{~nm}$ est la plus favorable, puisque les vitesses d'ablation sont environ deux fois plus élevées qu'à $248 \mathrm{~nm}$, et que la fluence utilisable est limitée par la sélectivité sur le conducteur inférieur (sans différence nette entre les deux longueurs d'ondes). De plus, les lasers fonctionnant à $308 \mathrm{~nm}(\mathrm{XeCl})$ sont plus faciles à conduire. 


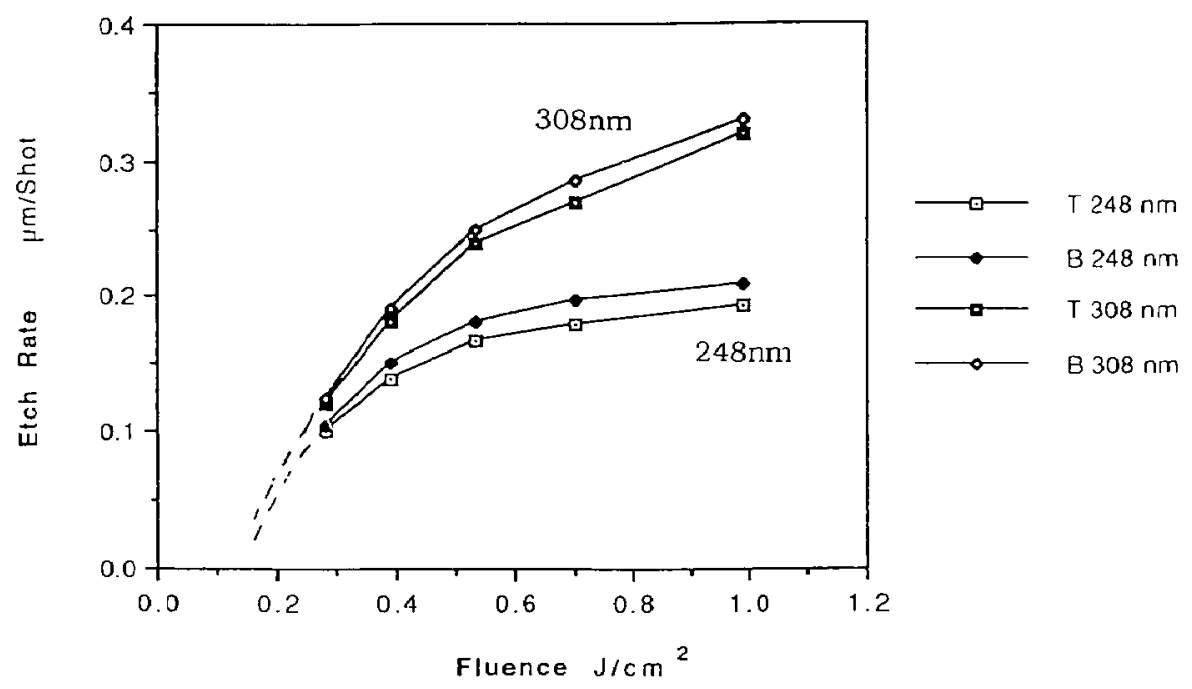

figure 5: Vitesses d'ablation en fonction de la fluence. $\mathrm{T}=$ "Thermid". $\mathrm{B}=$ benzocyclobutène.

La technique du masque métallique "in situ", souvent utilisée [11], est coûteuse en nombre d'étapes de procédé. Comme avec le laser $\mathrm{CO}_{2}$, nous avons donc choisi d'opérer en projection d'image trou par trou. Grâce à un travail sur le diaphragme projeté, nous savons réaliser, au choix, des trous à flancs raides ou des trous évasés à bords adoucis. C'est cette dernière forme que nous avons choisie. Elle permet de supprimer l'étape ultérieure de remplissage du trou et elle favorise un passage fiable du conducteur sur le rebord du trou (fig. 7).

\subsection{Développement d'une machine}

Une machine de perçage trou par trou a été développée, composée d'une source Excimère [12] et d'une station de travail placée en salle propre (fig. 6) [13].

Malgré l'utilisation de tables classiques, la sélection d'une tête laser tirant à $400 \mathrm{~Hz}$ et de la longueur d'onde $\mathrm{XeCl} 308 \mathrm{~nm}$ (environ 30 tirs par trou) autorise des cadences de perçage élevées: 250 secondes pour 1000 vias.

Le projecteur d'image permet d'ajuster le taux de réduction de 1 à 11 .

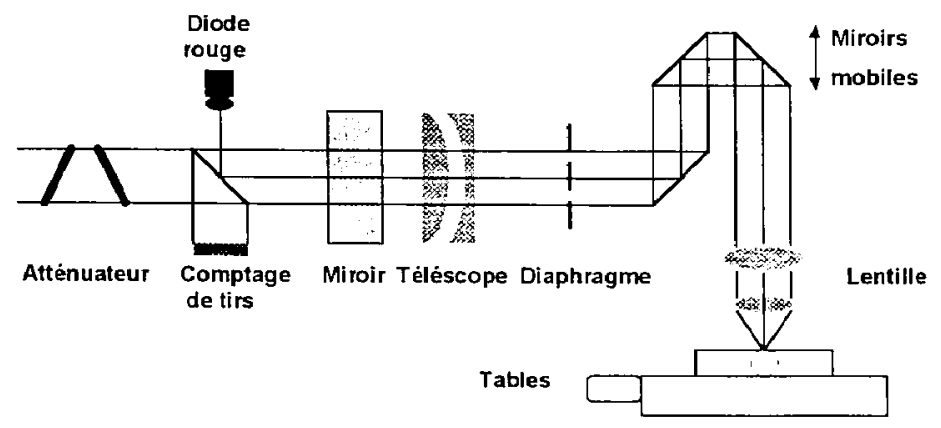

figure 6: Scliéma de la station de travail excimère 
En matière de productivité, le perçage laser procure de nouveau des gains appréciables. L'interfaçage avec la CAO permet la suppression des masques de photolithographie, et c'est de loin le procédé le plus économe en nombre d'étapes technologiques.

Notons que, dans cette conception, il existe encore des possibilités de gains ultérieurs de productivité, par division du faisceau et perçage simultanné de circuits multiples, et par alignement automatique des substrats.

\subsection{Résultats}

Ce matériel nous a permis de réaliser des trous évasés de $15 \mu \mathrm{m}$ d'ouverture avec une résistance de via $\leq 20 \mathrm{~m} \Omega$ (fig. 7). Les multicouches comportant de tels vias résistent aux essais climatiques (VRT $-55 /+125^{\circ} \mathrm{C}$, stockage $100 \mathrm{~h} 150^{\circ} \mathrm{C}$ ).

Le procédé de perçage est maintenant figé, il sera incorporé dans une technologie $\mathrm{MCM}$ très haute densité, encore en cours de développement (pour d'autres aspects) avec le soutien de la DRET.
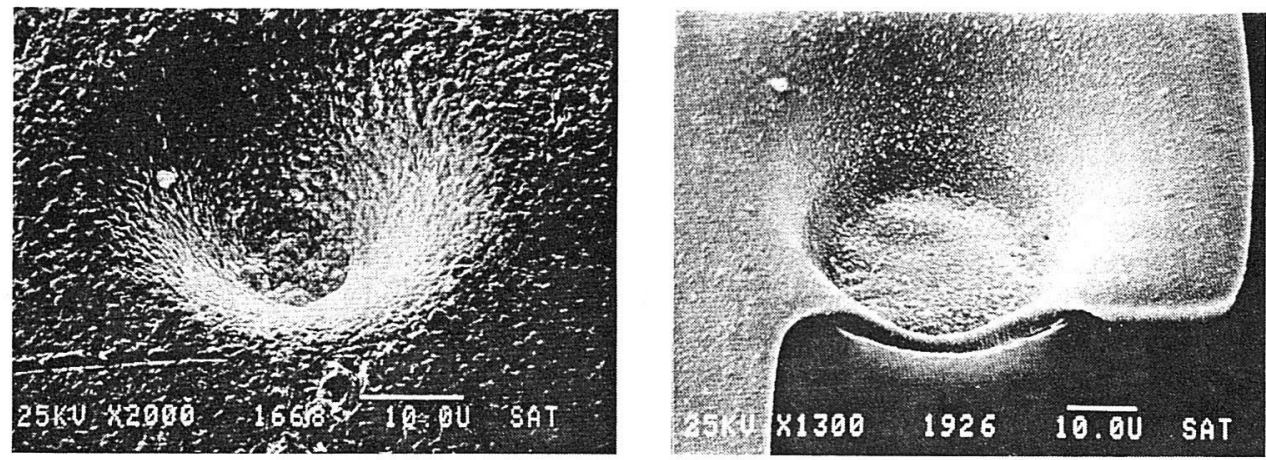

figure 7: Vias percés laser dans un diélectrique organique épaisscur $20 \mu m$. et métallisations conches minces.

\section{CONCLUSION}

Ces deux applications complètent la gamme des utilisations du laser en technologie micro-électronique (ajustage de résistances, prédécoupe de substrats céramiques, microsoudage, lentillage d'extrémités de fibres optiques, ...).

Nous avons poursuivi une double démarche. Confrontés à un même problème dans deux filières technologiques différentes, une analyse technico-économique nous à conduit à la sélection de deux types de laser différents: un laser infrarouge pour percer les diélectriques couches épaisses, et un laser UV pour percer les diélectriques organiques couches minces. Nous avons cherché à substituer les techniques laser aux anciennes sans augmentation des coûts de production.

Les techniques ont été validées sur des mires et deux machines de perçage ont été construites. Des applications sont en cours.

Développé à l'occasion de cette étude, le laser $\mathrm{CO}_{2}$ TEA à impulsion longue trouve d'autres applications dans le micro-usinage de matériaux fragiles et dans le domaine médical en odontologie, dermatologie, chirurgie. 
Dans les deux développements évoqués ici, nous avons trouvé chez nos partenaires toutes les compétences, tout le soutien et l'enthousiasme souhaitables. Nous les en remercions.

\section{Références:}

[1] Gilbert F., «Laser drilling interconnection enhanced substrates», IEEE Workshop on VLSI and microsystems packaging techniques and manufacturing technologies, Baveno, 6-7 Mai 1996.

[2] D'après Hass Opt. Soc. Am., 45, p 945 (1955), cité dans: Vannes A.B., Lasers et industries de transformation (CAST, INSA Lyon, 1986) p 113.

[3] Farcy J.Cl., Hélias G., «Le laser TEA dans les applications de micro-usinage industrielles et médicales», Journées d'information: Les applications du laser en micro-usinage, IREPA Laser, Strasbourg 24 Janvier 1996.

[4] Intégrateur: Baccini $\mathrm{SpA}$, Trévise.

[5] Hagan N.C. et al., The International Journal for Hybrid Microelectronics $12 \mathrm{n}^{\circ} 4$, (December 1989) 175-180.

[6] Massénat M., Multichip Modules d'hier et de demain (Polytechnica et Mentor Sciences, Paris, 1994) pp. 203-206.

[7] Messner G. et al., Thin Film Multichip Modules (ISHM Reston 1992) p 124.

[8] Rumsby P.T., Exitech Ltd, Oxford (1991), Etude commandée: le seuil de dommage sur or couches épaisses a été trouvé à $1 \mathrm{~J} / \mathrm{cm}^{2}$, environ.

[9] Witt M., Industrial application of excimer lasers, $p 421$.

[10] Tessier T.G., Chandler G., IEEE Trans. on Components, Hybrids and Mamufacturing Technology, 16 n$^{\circ} 1$, (February 1993) p 39.

[11] Brösamle H. et al., "Technology for a Microwiring Substrate», Siemens Research and Development Reports 5/88 17 n5 (1988) 213-262.

[12] LPX 240-i-CC, Lambda Physik, Göttingen.

[13] Conception et construction: Andrew R., Canivez Y., Optec S. A., Mons. 